\title{
La médecine de famille face à la dépression chronique
}

\author{
CLAIRE FITOUSSI $^{\mathrm{a}}$, AUDREY LINDER ${ }^{\mathrm{a}}$, Dr DANIEL WIDMER ${ }^{\mathrm{b}}$, Dr YVES DE ROTEN ${ }^{\mathrm{a}}$, Pr JEAN-NICOLAS DESPLAND $^{\mathrm{a}}$ et Dr GILLES AMBRESIN $^{\mathrm{a}}$
}

Rev Med Suisse 2020; 16: 1818-21

Cette étude s'intéresse à la prise en charge de la dépression chronique au cabinet du médecin de famille (MF) et à la collaboration MF-psychiatre. La pratique des MF de notre étude met en évidence deux situations différentes: les patients capables de verbaliser leur souffrance psychique et pouvant être directement référés au psychiatre et ceux exprimant leur souffrance psychique essentiellement par des symptômes physiques. Un travail de liaison psychique peut aider ces derniers à mettre en lien leurs symptômes somatiques et leur souffrance psychique et permettre de les référer au psychiatre. Si ce travail n'aboutit pas, le MF reste au premier plan de la prise en charge médicale. Un accompagnement sur le long terme se poursuit, où le MF en vient parfois à renoncer à guérir et à se concentrer sur la relation médecin-patient.

\section{Facing chronic depression in family practice}

This study focuses on the management of chronic depression at the general practitioner's office and the collaboration between general practitioner (GP)-psychiatrist. Our study's highlights two different situations: patients able to verbalize their psychological suffering and who can be directly referred to the psychiatrist and patients expressing their psychological suffering mainly by physical symptoms. GPs consider they first have to work with their patient to help them connect their somatic symptoms and their psychological suffering, which will allow them to refer their patient to the psychiatrist. If this work does not succeed, the GP remains at the forefront of medical care. Long-term support continues, where the GP sometimes ends up giving up on curing and focusing on the doctor/patient relationship.

\section{LA MÉDECINE DE FAMILLE FACE À LA DÉPRESSION CHRONIQUE}

En Suisse, la prévalence pour la vie entière de la dépression chronique est estimée entre $6^{1}$ et $15 \% .^{2}$ Selon l'Observatoire suisse de la santé (OBSAN), ${ }^{3}$ les médecins de famille (MF) posent le diagnostic de $41 \%$ des dépressions, le reste d'entre elles étant diagnostiquées par le psychiatre. Les MF estiment que la moitié des premières consultations pour dépression concernent des dépressions récurrentes, voire chroniques.

a Institut universitaire de psychothérapie, DP-CHUV et UNIL, Bâtiment les Cèdres, Site de Cery, 1008 Prilly, ${ }^{b}$ Médecine psychosomatique et psychosociale ASMPP, Département de médecine de famille, Unisanté, 1011 Lausanne

fitoussiclaire@gmail.com | audrey.linder@chuv.ch |drdanielwidmer@hin.ch yves.deroten@chuv.ch | jean-nicolas.despland@chuv.ch

gilles.ambresin@chuv.ch
Toujours selon l'OBSAN, les MF recourent à un traitement exclusivement médicamenteux dans $60 \%$ des dépressions légères et $50 \%$ des dépressions moyennes. Pour les dépressions sévères, l'approche combinée, par antidépresseurs et psychothérapie, est privilégiée. De plus, 20 à $40 \%$ des personnes souffrant de problèmes psychologiques ne consultent que le MF et manifestent leur souffrance psychique à travers des symptômes somatiques ou sous forme de comorbidités somato-psychiques. ${ }^{4}$ Ces chiffres soulignent l'importance d'une prise en charge somato-psychique et d'une collaboration entre les MF et les psychiatres. De fait, les MF offrent fréquemment un soutien psychologique ${ }^{5}$ et, depuis 1999 en Suisse romande, un Certificate of Advanced Studies (CAS) leur permet de développer des compétences pour une prise en charge psychosociale et psychosomatique. Ce certificat permet d'obtenir des crédits pour le certificat de formation complémentaire en médecine psychosomatique et psychosociale de la $\mathrm{FMH} .{ }^{6}$ Quant à la collaboration entre MF et psychiatres, plusieurs études évoquent une méconnaissance réciproque et un manque de clarté sur le rôle de chacun. ${ }^{4,7}$

Dans ce contexte, nous avons constitué un groupe de recherche pluriprofessionnel (psychiatre, psychologue, médecin de famille, sociologue et étudiante en médecine) et mené une étude qualitative par Focus Groups (FG). Notre question de recherche portait sur trois axes: les représentations de la dépression chronique par les MF, sa prise en charge, ainsi que la collaboration MF-psychiatres (voir ci-dessous). Dans cet article, nous ne traitons pas en détail des représentations de la dépression chronique par les MF, ce sujet ayant fait l'objet d'un précédent article. ${ }^{8}$

\section{Méthodologie de l'étude}

Étude qualitative, menée par un groupe de travail pluriprofessionnel, sur les représentations et la prise en charge de la dépression chronique par les médecins de famille ainsi que leur collaboration avec les psychiatres. L'étude est basée sur trois Focus Groups (FG) de 4, 7 et 11 participant.e.s, tou.te.s médecins de famille en Suisse romande. Ils ont été constitués selon un échantillonnage raisonné valorisant la diversité des médecins en termes d'âge, de sexe, de lieu d'installation (ville-campagne) et de formation (CAS ou non), et ont été menés selon une grille de questions ouvertes avec des questions de relance. Ils ont été retranscrits, codés, ${ }^{8}$ puis une carte conceptuelle a été élaborée à partir des codes regroupés en thématiques (une carte conceptuelle s'élabore également à partir des mémos analytiques; ces derniers sont des commentaires associés aux verbatim, aux codes ou aux thèmes, sont élaborés tout au long du processus de codage et constituent un journal d'enquête). La carte conceptuelle a été établie par 
quatre des auteurs (AL, CF, DW et GA) à partir de leurs mémos analytiques puis retravaillée avec l'ensemble de l'équipe de recherche.

\section{RÉSULTATS}

L'analyse des FG nous a permis d'élaborer une carte conceptuelle (figure 1) qui modélise le travail des MF dans le traitement de la dépression chronique, ainsi que leur collaboration avec les psychiatres. La partie haute de la carte conceptuelle dépeint les cas où le $\mathrm{MF}$ accueille à son cabinet des patients souffrant de dépression chronique disposés à l'introspection. Les MF les décrivent comme des «bons cas» pour le psychiatre et les réfèrent directement, invoquant parfois de surcroît le manque de temps. De fait, ces patients acceptent volontiers le recours au psychiatre.

«C'est des gens qui sont capables justement de lire au fond d'euxmêmes leurs émotions, d'élaborer, de faire des liens, c'est le profil plutôt intellectuel, si on veut bien. J'aurais tendance à envoyer plutôt ces patients chez le psychiatre.» (Extrait d'un FG)

La suite de la carte conceptuelle décrit les cas des patients souffrant de dépression chronique qui expriment leur souffrance psychique essentiellement par des symptômes physiques. Á ce stade, le MF peut recourir au spécialiste pour établir le diagnostic ou pour l'introduction d'un traitement médicamenteux. Toutefois, il peut aussi considérer que son patient ne fait pas partie des «bons cas» à envoyer au psychiatre, ou du moins pas encore. Au travers du lien thérapeutique, le MF prépare alors le patient à une prise en charge psychothérapeutique en élaborant la plainte somatique. Lorsque son patient est prêt pour une démarche introspective approfondie, il le réfère au psychiatre. «ll faut des années avant que petit à petit le matériel émotionnel émerge puis qu'on puisse commencer au travers du lien à faire émerger des choses, et puis mon expérience c'est que souvent, à certains moments, (...) j'ai l'impression que c'est «bingo», c'est quand les gens peuvent être branchés sur la prise en charge psychothérapeutique. Tout d'un coup, les éléments sont là et puis ils peuvent passer à la vitesse supérieure.» (Extrait d'un FG)

En revanche, si la dépression chronique continue de s'exprimer essentiellement par des symptômes du corps, le MF ne réfère pas le patient et un travail de longue haleine se poursuit. Le MF affronte ses propres sentiments de solitude et d'impuissance qu'il gère en «s'occupant de lui-même»; il intègre alors des groupes Balint, recourt à des supervisions et collabore avec un réseau de soins impliquant des intervenants supplémentaires (infirmières, ergothérapeutes, CMS, etc.).

Dans la prise en charge, le MF pratique en premier lieu des actions de guérison (cure). Dans un second temps, il peut percevoir que son agir est une réponse à son sentiment d'impuissance. Cette prise de conscience le conduit à renoncer à guérir pour être dans une démarche de soins (care), d'accompagnement.

«ll y a une espèce d'acceptation avec ces dépressifs chroniques finalement, on les accompagne, quoi. Enfin, à un moment donné, on arrête d'essayer de vouloir à tout prix les guérir, il me semble.» (Extrait d'un FG)

Dans ces cas, le MF, supervisé ou non, est le seul médecin au sein du réseau et assume la responsabilité médicale de la prise en charge. Même s'il considère que son patient n'est pas un bon candidat pour le psychiatre, les portes de la collaboration

\begin{tabular}{l|l|c|l}
\hline & \multirow{2}{*}{ FIG 1} & $\begin{array}{c}\text { Carte conceptuelle : le travail des médecins de famille dans } \\
\text { la prise en charge de la dépression chronique }\end{array}$ & \\
\hline
\end{tabular}

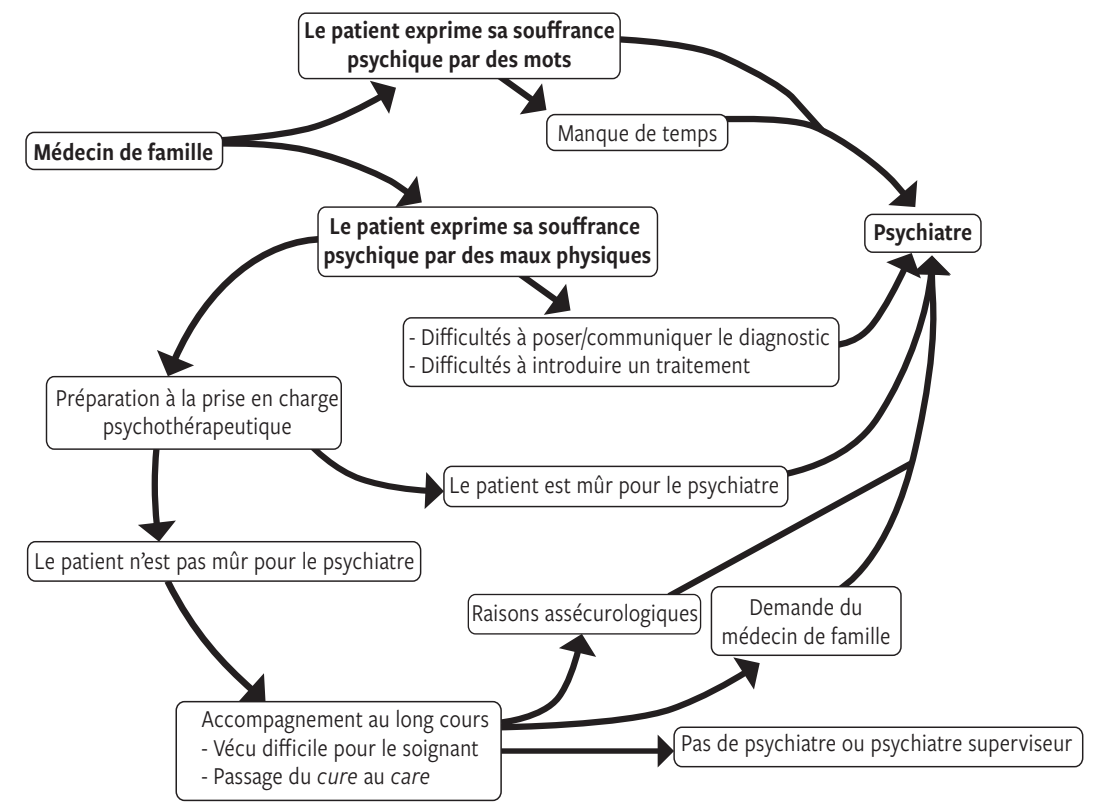


ne sont pas fermées pour autant. Le psychiatre entre en scène lorsque, en raison d'arrêts de travail, surviennent des demandes de l'employeur ou de l'assurance (expertises). En outre, le MF contacte le psychiatre lorsqu'il sent que la situation dépasse ses compétences ou sa disponibilité en temps.

«On fait pas juste quand on n'a pas le temps de remonter à des choses importantes. [...] Mais on n'a pas assez de temps parce qu'on a tous trop de patients. C'est un peu ça le problème. [...] Parce qu'on n’a pas le temps, même si on en a envie.» (Extrait d'un FG)

Une fois chez le psychiatre, le patient souffrant de dépression chronique peut continuer à consulter son MF en parallèle. Dans ce cas, le manque de clarté de son rôle et de celui du psychiatre oblige le MF à s'adapter, selon que le psychiatre se charge ou non de la médication et des arrêts de travail.

«On doit être souple, comme généralistes.» (Extrait d’un FG)

\section{DISCUSSION}

\section{Orientations du traitement}

Les MF de notre étude identifient un groupe de patients comme disposés à l'introspection et les orientent directement vers la psychothérapie. Le terme de YAVIS (Young, Attractive, Verbal, Intelligent and Successful) désigne un profil de personnes considérées comme «idéales pour la psychothérapie». ${ }^{9}$ Dès lors, les thérapeutes seraient plus confiants dans l'efficacité du traitement lorsqu'ils ont affaire à ce type de personnes et s'impliqueraient également davantage. ${ }^{9}$ Ce biais positif, mis en évidence par Schofield, semble partagé par les MF de notre étude, qui considèrent que les YAVIS s'engagent davantage dans la psychothérapie. ${ }^{9}$

Une inégalité sociale d'accès à la psychothérapie a été révélée par des études françaises, qui montrent que les personnes d'un niveau d'éducation élevée ont davantage accès aux psychothérapies que les personnes de faible statut socio-économique. ${ }^{5,10}$ On peut penser que les représentations du MF liées au syndrome YAVIS jouent un rôle dans cette inégalité.

Dans les situations où les plaintes somatiques prédominent, une préparation à la prise en charge psychothérapeutique est évoquée par certains MF. Le terme de «travail de liaison psychique» correspond au travail préparatoire qui permet un accès plus facile aux soins psychiatriques ${ }^{4}$ et qui est possible grâce à la confiance entre le médecin et son patient. Cette liaison «réintroduit le patient en tant que sujet de son histoire, et non plus seulement comme demandeur de prestations».4

\section{Accompagnement sur le long terme}

Les MF de notre étude perçoivent la nécessité d'un accompagnement au long cours des patients souffrant de dépression chronique. Pour s'y inscrire, ils sont amenés à renoncer à l'agir médical à visée de guérison.

Etre en relation avec la souffrance des autres a un impact sur le psychisme des soignants. «Dans certaines situations, le patient ne va pas se contenter d'exprimer sa souffrance par une plainte physique ou morale. Il va, par (un) mécanisme psychique assez archaïque, faire vivre à ses soignants un malaise intense, gênant, prenant et assez inexplicable, en tout cas en apparence (...). La communauté de symptômes installée va plonger le soignant dans le même vécu de débordement psychique incontenable que celui qu'éprouvait, ou qu'éprouve, le patient. »" Par ce mécanisme, les soignants sont poussés à «répondre dans le registre de l'agir (...) afin de réduire l'anxiété que provoque l'impossibilité d'élaborer ce qui les envahit»." Les médecins pourraient utiliser leur vécu de la consultation pour mieux comprendre ce que vivent les patients, avant de se laisser aller à répondre par l'agir.

Nous pouvons également explorer l'agir médical en suivant Balint, pour qui la rencontre à l'autre n'est possible que lorsque le médecin prend conscience de sa fonction apostolique: «Tout se passe comme si chaque médecin avait la connaissance révélée de ce qui est juste et faux que son patient attende et supporte. Et au-delà, comme s'il avait un devoir sacré de convertir à sa foi toute la part d'ignorance et d'incroyance de son patient. $\gg^{12}$ Prendre conscience de ce besoin d'agir et trouver un nouvel équilibre pour travailler sur le registre de la relation, permet au MF de passer des actes de guérison aux soins, du cure au care.

On peut enfin s'interroger si la conviction des MF que certains patients ne sont pas susceptibles d'entreprendre une psychothérapie relève de cette fonction apostolique, mais qui, cette fois, les pousserait précisément à ne pas agir.

\section{Collaboration avec les psychiatres}

Nos FG relèvent l'opacité des rôles respectifs dans la collaboration MF-psychiatres. Un article publié en 2011, basé sur deux sondages auprès d'une centaine de psychiatres et d'une centaine de MF belges, insiste sur la nécessaire collaboration entre MF et psychiatres. Toutefois, les sondages réalisés montrent que l'image que les MF ont des psychiatres et vice versa n'est de loin pas toujours positive et qu'il existe une méconnaissance des spécificités du travail de chacun. ${ }^{7}$ Cette méconnaissance et le manque de clarté sur les rôles respectifs illustrent l'importance de la complémentarité et de l'échange entre MF et psychiatres pour assurer un système de santé mentale plus efficace. ${ }^{4}$ Il nous semble qu'une des voies possibles serait d'associer plus communément et précocement un psychiatre de premier recours dans la prise en charge, en l'intégrant dans un même centre de soins primaires que le MF. ${ }^{13}$ Ce psychiatre aurait des compétences dans l'évaluation et la prise en charge brèves d'un large éventail de troubles mentaux courants et pourrait assurer la supervision d'autres collègues offrant ces soins, ainsi que des consultations avec eux.

\section{CONCLUSION}

Les MF ont un rôle central dans la prise en charge de la dépression chronique, en particulier lorsque la souffrance psychique est exprimée essentiellement au travers des symptômes physiques. Un travail de liaison psychique est nécessaire avant que le patient puisse être référé à un psychiatre, car il permet de mettre en lien les symptômes somatiques 
avec la détresse psychologique. Lorsque ce travail n'est pas possible ou tant qu'il n'aboutit pas, les MF poursuivent leur accompagnement sur le long terme. Dans le cadre de ce suivi, les MF peuvent déployer leurs compétences avec l'aide d'intervisions par des groupes de pairs, du CAS en médecine psychosomatique et psychosociale et de supervisions chez un spécialiste en santé mentale. Ces recours sont-ils suffisamment exploités?

Conflit d'intérêts: Les auteurs n'ont déclaré aucun conflit d'intérêts en relation avec cet article.

\section{IMPLICATIONS PRATIQUES}

- Les médecins de famille (MF) décrivent deux types de patients souffrant de dépression chronique: les patients qui verbalisent leur souffrance psychique et ceux qui l'expriment essentiellement par des symptômes somatiques. Pour une partie de ces derniers, un travail de liaison psychique prodigué par le MF permet une préparation à la prise en charge par le psychiatre

- Un accompagnement sur le long terme est prodigué par les MF lorsque les symptômes restent exprimés essentiellement par le corps. Des formations complémentaires, des supervisions chez des psychiatres ou psychothérapeutes ainsi que des intervisions constituent des aides précieuses pour les MF

- Pour la prise en charge de ce type de patients, qui présentent à la fois des symptômes physiques et psychiques, de nouveaux modèles, qui sont à développer, intégreraient la prise en charge de la santé mentale dans les centres de soins primaires

\footnotetext{
1 Angst J, Gamma A, Rössler W, et al. Long-term depression versus episodic major depression : Results from the prospective Zurich study of a community sample. J Affect Disord 2009;115:112-21.

2 Vandeleur CL, Fassassi S, Castelao E, et al. Prevalence and correlates of DSM-5 major depressive and related disorders in the community. Psychiatry Res

2017;250:50-8.

3 Baer N, Schuler D, Füglister-Dousse S, et al. La dépression dans la population suisse : données concernant l'épidémiologie, le traitement et l'intégration
}

socioprofessionnelle. Neuchâtel : Observatoire suisse de la santé. 2013;56:140.

$4{ }^{*}$ Guex $P$, Barbier Y. Le généraliste et le psychiatre : échec ou réussite ? Rev Med Suisse 2005;1:2136-41.

5 *Dumesnil H, Cortaredona S, Cavillon $M$, et al. Prise en charge de la dépression en médecine générale de ville. Résultats issus d'une enquête menée auprès d'un panel national. Inf Psychiatr 2014;90:350. 6 ISFM. Médecine psychosomatique et psycho-sociale. (ASMPP) 2004;1-8. 7 *Philippe P. Généralistes - Psychiatres
: amis ou ennemis? Rev Méd Liège 2011;66:92-101.

8 Linder A, Widmer D, Fitoussi C, et al.

Les médecins généralistes face à la

« dépression chronique ». Représenta-

tions et attitudes thérapeutiques. RFAS 2018:4:329-58

9 Schofield W. Psychotherapy : the purchase of friendship. Piscataway :

Transaction Publishers. 1986.

10 Briffault X, Sapinho D, Villamaux M, et al. Factors associated with use of psychotherapy. Soc Psy and Psychiatric Epidemiol 2008;43:165-71.
11 Morasz L. Prendre en charge la souffrance à l'hôpital: patients, soignants, familles. Malakoff : Éditions Dunod, 2003. 12 Balint M. Le médecin, son malade et la maladie. Lausanne : Payot, 1996.

13 Gask L, Khanna T. Ways of working at the interface between primary and specialist mental healthcare. $\mathrm{Br} \mathrm{J}$ Psychiatr 2011;198:3-5.

** à lire 